\title{
CUSA and CUDE: GPU-Accelerated Methods for Estimating Solvent Accessible Surface Area and Desolvation
}

\author{
DAVID DYNERMAN,${ }^{1}$ ERICK BUTZLAFF ${ }^{2}$ and JULIE C. MITCHELL ${ }^{3}$
}

\begin{abstract}
It is well-established that a linear correlation exists between accessible surface areas and experimentally measured solvation energies. Combining this knowledge with an analytic formula for calculation of solvent accessible surfaces, we derive a simple model of desolvation energy as a differentiable function of atomic positions. Additionally, we find that this algorithm is particularly well suited for hardware acceleration on graphics processing units (GPUs), outperforming the CPU by up to two orders of magnitude. We explore the scaling of this desolvation algorithm and provide implementation details applicable to general pairwise algorithms.
\end{abstract}

Key words: desolvation, GPU computing, NVIDIA CUDA, protein docking, solvent accessible surface area (SASA).

\section{INTRODUCTION}

$\mathbf{T}$ HE PREDICTION OF THE BOUND CONFIGURATION OF TWO PROTEINS is an important problem in computational biology. One approach involves defining and globally minimizing a function that describes the various energetic interactions between intermolecular pairs of atoms (Totrov et al., 1997; Rarey et al., 1996; Caflisch et al., 1992; Given et al., 1998; Goodsell et al., 1990; Hart et al., 1992; Mitchell et al., 1999; Marcia et al., 2005; Klepeis et al., 1998; Gray et al., 2003). One way to account for the hydrophobic effect in such a function is to exploit the strong linear relationship between accessible surface area and the desolvation energy of binding. Energetic methods attempt to define and optimize functions for which the native solution produces the global minimum energy. In addition to terms describing electrostatic and van der Waals interactions, the inclusion of a desolvation term can significantly improve predictions (Camacho et al., 1999; Horton et al., 1992; Eisenberg et al., 1989; Cummings et al., 1995; Eisenberg et al., 1986; Juffer et al., 1995).

Most global optimization algorithms require many evaluations of the associated energy function. Thus, it is crucial that the function evaluation be as efficient as possible. Graphics processing units, referred to as GPUs, are emerging as high-performance parallel coprocessors able to greatly speed certain types of calculations. This paper describes our efforts at developing CUSA and CUDE, codes that compute solvent

\footnotetext{
${ }^{1}$ Department of Mathematics, ${ }^{2}$ Department of AMEP and Economics, and ${ }^{3}$ Departments of Mathematics and Biochemistry, University of Wisconsin, Madison, Wisconsin.
} 
accessible surface area and desolvation using the CUDA programming language (NVIDIA, 2007), which will be described in Section 2.2.2.

\subsection{Solvent accessible surface area}

Many different approaches have been applied to the approximation of the accessible surface area of Lee and Richards, which in concept rolls a sphere of average solvent radius over the surface of a macromolecule. The solvent probe's boundary traces out the molecular surface, while its center traces out the solvent accessible surface (Lee et al., 1971).

The solvent accessible surface area (SASA) method of Hasel et al. that we implement is one of a class of pairwise, twice differentiable approximate algorithms for computing SASA developed by the group of W. Clark Still (Weiser et al., 1999a,b,c). A later method in this class - the method of linear combinations of pairwise overlaps (LCPO) of Weiser et al. (1999a) - utilizes more parameters than the algorithm we implement, and to improve runtime performance, it uses a pre-computed list of atomic overlap information. These neighbor lists are compiled through the application of an atomic model based on Gaussian distributions (Weiser et al., 1999b).

Exact methods for the rapid calculation of SASA are also widely available. The first differential geometrybased analytic model published by Connolly (1983) has been made more efficient (Fraczkiewicz et al., 1998; Sanner et al., 1996). As a benchmark for our own SASA calculations we have used NACCESS, a near-exact method for computing SASA that slices the macromolecule to obtain 2D exterior boundaries, adds the solvent probe radius to the boundaries of each slice, and interpolates the surface between slices to compute SASA (Hubbard et al., 1993).

Because our goal is to devise a surface area calculation that is ideal for GPU computing, we avoid models having a large number of parameters or which implement inhomogeneous calculations. By applying a pairwise model with a minimal set of atom parameters, we achieve up to two orders of magnitude speedup. Moreover, the model is twice differentiable, making it well-suited for use in optimization schemes. These requirements led us to choose the SASA model of Hasel et al. (1988), which is described in Section 1.1.2.

1.1.1. Buried surface area. The buried surface area (BSA) of a complex between two bound proteins is the total surface area of both proteins that is removed from solvent upon binding. If the proteins have individual surface areas $\mathrm{A}_{1}$ and $\mathrm{A}_{2}$, and if the area of the complex itself exposed to solvent is $A_{C}$, then

$$
A_{1}+A_{2}=A_{\mathrm{C}}+\mathrm{BSA}
$$

Rearranging this relationship gives a convenient formula for buried surface area:

$$
\mathrm{BSA}=A_{1}+A_{2}-A_{\mathrm{C}} .
$$

Rewriting the formula for BSA in terms of per atom changes in solvent accessibility, we see that

$$
\mathrm{BSA}=\sum_{i=1}^{N_{1}}\left(-\Delta A_{i}\right)+\sum_{j=1}^{N_{2}}\left(-\Delta A_{j}\right)
$$

The negative signs arise due to the fact that BSA is a positive quantity, while $\Delta \mathrm{A}_{\mathrm{i}}<0$ for atoms in the interface.

In this study we use buried surface area to fit the parameters of the SASA algorithm of Hasel et al. (1988). We use the computed areas along with the Eisenberg-McLachlan atomic solvation parameter set (Eisenberg et al., 1986) to compute binding desolvation energy.

1.1.2. Pairwise accessibility calculation. A key element of our desolvation energy algorithm is the geometrically intuitive method of Hasel et al., for approximating the accessible surface area of Lee and Richards (Hasel et al., 1988; Lee et al., 1971). They showed that a good approximation to the SASA of atom $i$ in a protein of $N$ atoms is

$$
A_{i}=S_{i}\left(\prod_{j}^{N} f_{i j}\right)
$$


where

$$
\begin{gathered}
f_{i j}=1-\frac{p_{i} p_{i j} b_{i j}}{S_{i}} \\
S_{i}=4 \pi\left(r_{i}+r_{s}\right)^{2} \\
b_{i j}=\pi\left(r_{i}+r_{s}\right)\left(r_{i}+r_{j}+2 r_{s}-d_{i j}\right)\left(1+\frac{r_{j}-r_{i}}{d_{i j}}\right)
\end{gathered}
$$

Intuitively, $S_{i}$ is the SASA of atom $i$ in the absence of overlaps, and $b_{i j}$ is the factor for reduction in surface area due to atom $j$ overlapping with atom $i$. However, the SASA reduction from multiple overlaps is not additive, so $p_{i}$ is an atom-wise parameter used to reduce the magnitude of $b_{i j}$ across multiple overlaps. Finally, $p_{i j}<1$ is a parameter dependent on whether or not atoms $i$ and $j$ are covalently bound. In the auxiliary formulas, $r_{i}$ is the radius of atom $i, r_{s}$ is the radius of the solvent probe whose center traces out the accessible surface (typically $r_{s}=1.4 \AA$ is used), and $d_{i j}$ is the distance between atoms $i$ and $j$.

Equation (4) allows us to compute the change in SASA during binding of proteins $P_{1}$ and $P_{2}$ as

$$
\Delta A_{i}=S_{i}\left(\prod_{j=1}^{N_{1}} f_{i j}\right)\left(\prod_{k=1}^{N_{2}} f_{i k}\right)-A_{i, \text { initial }}
$$

where

$$
A_{i, \text { initial }}=S_{i}\left(\prod_{j=1}^{N} f_{i j}\right)
$$

for $N=N_{1}$ or $N=N_{2}$, depending on whether atom $i$ belongs to $P_{1}$ or $P_{2}$ respectively.

Note that to allow for flexible docking, formula (8) cannot be easily simplified. In this equation, the products of $f_{i j}$ and $f_{i k}$ terms represent the atomic overlap after binding, while the product of $f_{i j}$ 's included in the $\mathrm{A}_{i \text {,initial }}$ term represents overlap before binding. Conformational changes during binding imply that these terms will generally not be equal.

\subsection{Desolvation energy}

The linear relationship demonstrated by Eisenberg and McLachlan (1986) between the accessible surface area of Lee and Richards (1971) and experimentally determined solvation energy of transfer provides a way to approximate hydrophobic effects. More recent work (Wagoner et al., 2006) shows that introducing solvent accessible volume information improves model accuracy at the cost of more complex computations.

Eisenberg and McLachlan showed that, for a solvated protein consisting of $N$ atoms, the energy of solvation can be estimated as:

$$
\Delta G_{\text {solv }}=\sum_{i=1}^{N} \gamma_{i} A_{i}
$$

The $\gamma_{i}$ are constant values that depend on atom type. These atomic solvation parameters, or ASPs, are frequently written as $\Delta \sigma_{i}$, as they represent the Gibbs free energy $\Delta G$ associated with the solvation of individual atoms.

In this work, we use formula (10) to characterize the energetic contribution of the hydrophobic effect in protein binding:

$$
\Delta G_{\text {bind,solv }}=\sum_{i=1}^{N_{C}} \gamma_{i} \Delta A_{i}
$$

Here, $\Delta G_{\text {bind,solv }}$ describes the energetic effect of desolvating the atoms at the active sites of the proteins during binding, and $\Delta A_{i}$ can be computed from formula (8). This sum is taken over all atoms in the proteinprotein complex of $N_{C}$ total atoms - that is, if the individual proteins are comprised of $N_{1}$ and $N_{2}$ atoms, then 


$$
\begin{aligned}
\Delta G_{\text {bind,solv }} & =\sum_{\mathrm{i}=1}^{N_{C}} \gamma_{i} \Delta A_{i} \\
& =\sum_{i=1}^{N_{1}} \gamma_{i} \Delta A_{i}+\sum_{j=1}^{N_{2}} \gamma_{j} \Delta A_{j} .
\end{aligned}
$$

Note that the change $\Delta A_{i}$ in area for atom $i$ may be affected by conformational changes in addition to binding events.

1.2.1. Atomic solvation parameters. Many sets of ASP values $\gamma_{i}$ have been published. Juffer et al. (1995) provide an overview and comparison of several such sets. This study found poor and often contradictory performance among various ASP sets. They suggest that the Eisenberg ASPs (Eisenberg et al., 1986) are most consistent in systems with strong hydrophobic interactions which led to their use in our study.

In 1995, Cummings et al. (1995) presented a comprehensive study of ASPs derived for use in protein docking simulation. The study took three widely used SASA sets-those reported in Eisenberg et al. (1989); Wesson and Eisenberg (1992); and Lesser and Rose (1990) — and regressed them on three sets of transfer energy data: those of Fauchere and Pliska (1983); Radzicka and Wolfenden (1988); and Wolfenden et al. (1993). Of these pairings, they found that the ASP set that best discriminated between correct and incorrect protein docking results as a complement to their own energy function-comprised of an electrostatic and Lennard-Jones potential - was that derived from the regression of the SASA set of Lesser and Rose on the transfer energy set of Fauchere and Pliska (Cummings et al., 1995).

A more recent study by Zhou and Zhou (2002a,b) takes an entirely different approach to deriving ASPs. They derive a mutation experiment-based amino acid residue stability scale to determine the degree to which various residues are hydrophobic, and show that the experimentally determined free transfer energy of the most hydrophobic portion of this scale has excellent correlation with octanol-to-water transfer free energy. ASPs for the atoms in these hydrophobic residues are taken from the correlation (Zhou and Zhou, 2002a,b).

\subsection{Computation on GPUs}

Researchers have exploited graphics hardware for general purpose scientific computation since the 1970s (Owens et al., 2007). Within the past half decade, commodity desktop graphics processing units (GPUs) have become progressively more capable, most importantly in terms of programmability and key hardware features necessary for general purpose GPU, or GPGPU, computation (Owens et al., 2008).

Initially, programmable GPUs had to be accessed through a graphics programming library such as OpenGL or DirectX. Use of these complex libraries required a substantial graphics programming background. Particularly laborious was the reinterpretation of a nongraphics problem in the language of $3 \mathrm{D}$ visualization, and a significant amount of work was invested into understanding how fundamental scientific computing algorithms, such as general matrix/vector arithmetic, map to the GPU (Krüger and Westermann, 2005). These early results were promising, but they also required significant technical skill and impressive knowledge of graphics programming. For instance, Krüger and Westermann (2005) discusses the performance benefits of mapping a vector to a 2D texture, and Yang et al. (2007) describe the efficiency improvements of using OpenGL frame buffer objects.

For our work, the most significant advancement in GPU computing is the recent proliferation of software tools (and accompanying hardware support) targeted at easing GPGPU programming (NVIDIA, 2007; ATI, 2007; Buck et al., 2004). These tools greatly simplify scientific computing on GPUs, all but eliminating the type of requisite graphics programming knowledge described in the above referenced literature. However, as we discuss in Section 3.3, GPUs are not always the answer-care is required to design GPU algorithms for optimal scaling and speedup.

Several recent articles describe the evolution and technical implications of modern GPU computing in greater detail (Owens et al., 2007, 2008; Stone et al., 2007).

\section{METHODS}

We develop a pairwise parametric atomic algorithm for computing desolvation energy based on the algorithm for accessible surface area of Hasel et al. (1988) and the energy of solvation of Eisenberg and 
McLachlan (1986). To address the computational complexity of the accessibility calculation, we use the CUDA language for NVIDIA graphics processing units (GPUs) to greatly speed up batched desolvation calculations. The resulting CUDA algorithms for solvent accessible surface area (CUSA) and desolvation (CUDE) are well-suited for applications in computational structural biology.

\subsection{CUSA and CUDE models}

The solvation energy model proposed by Eisenberg and McLachlan (1986) provides atomic solvation parameters for five groups of atoms: $\gamma_{C}=30.5, \gamma_{S}=21.0, \gamma_{N / O}=-0.9, \gamma_{N+}=-38.5$, and $\gamma_{O-}=-15.0$, all in units of cal $\AA^{-2} \mathrm{~mol}^{-1}$. These values were computed from a linear least-squares fit of equation (10) against experimentally determined transfer free energies taken from Fauchere and Pliska (1983) and Eisenberg and McLachlan (1986). The SASAs used in this regression were computed by Shrake and Rupley (1973) using the atomic radii of Pauling (1960). To remain consistent with these varied initial studies, we use a united atom model with radii taken from Pauling (1960).

The desolvation model outlined above can be implemented as a simple algorithm that first computes solvent accessibility and then desolvation energy. The accessibility computation must iterate over each pair of atoms in the complex, combining $p_{i}$ parameters with separation information to obtain the per atom accessible surface areas. This per-atom accessibility information is then scaled by the relevant ASP values and summed to complete the desolvation calculation.

Using accessible surface area to calculate the solvation energy in this fashion is computationally expensive. This cost is further compounded in a minimization setting, where many function evaluations may be required to achieve convergence to a local or global minimum. The most expensive portion of the outlined algorithm is the accessibility calculation, which requires a pairwise comparison of all atoms in a complex and scales as $O\left(\left(N_{1}+N_{2}\right)^{2}\right)$. Furthermore, since SASA is a function of atomic positions, a minimization application must ensure all accessibilities are properly updated at each iteration. Reducing the runtime cost of the desolvation calculation will allow a minimization algorithm to more rapidly predict near-native structures.

2.1.1. Solvent accessible surface model. The method of Hasel et al. described in Section 1.1 .2 provides $p_{i}$ parameters and atomic radii for a united atom model that includes polar hydrogens. Atoms are grouped into 23 types according to hybridization and binding partners. In order to align the SASA algorithm with the united atom model dictated by the ASPs of Eisenberg and McLachlan (1986), we reduce the number of atom types to 12, excluding parameter values for polar hydrogens, carbon atoms with hybridization types not commonly found in proteins, and the elements $\mathrm{F}, \mathrm{Cl}, \mathrm{Br}$, and I, which do not occur in the systems we study. We perform a new fit of the $p_{i}$ values to minimize the square error over a benchmark set of $M$ known receptor/ligand complexes. The optimization of the parameters is described succinctly as:

$$
p_{i}^{*}=\underset{p_{i}}{\operatorname{argmin}} \sum_{j=1}^{M}\left(\mathrm{BSA}_{j}^{\mathrm{NACC}}-\mathrm{BSA}_{j}^{\mathrm{CUSA}}\left(p_{i}\right)\right)^{2}
$$

For this new regression, we use the Mintseris et al. (2005) protein docking benchmark. Benchmark files in PDB format (Berman et al., 2000) were obtained from the Weng group's website. 1ML0 was excluded from the benchmark because the available complex PDB file did not include all the chains present in the Mintseris et al. (2005) PDB files.

For each known complex in the training set, we used NACCESS (Hubbard et al., 1993) to estimate the buried surface area $B S A^{\mathrm{NACC}}$ from equation (2). We then iterated each of the $12 p_{i}$ 's individually over the range $0<p_{i}<5$ and computed $B S A^{\mathrm{CUSA}}\left(p_{i}\right)$ at each step using equations (3) and (8). The search interval is based on the original $p_{i}$ values derived by Hasel et al., In our search, we first used a coarse-grain step size of 0.1 and then performed a second refined search using step sizes of 0.001 in the $[x-0.1, x+0.1]$ interval around the coarse-grain minimum $x$. The resulting values are the new $p_{i}^{*}$ parameters (Table 1) that approximately minimize equation (13).

Additionally, the $p_{i j}$ factor that appears in formula (5) is based on whether atoms $i$ and $j$ are covalently bound (Hasel et al., 1988). Hasel et al. (1988) set this value to $p_{i j}=0.8875$ if atoms $i$ and $j$ are covalently bound, and $p_{i j}=0.3516$ otherwise. To simplify our calculations, we set $p_{i j}=0.8875$ if atoms $i$ and $j$ are within $1.8 \AA$ and $p_{i j}=0.3516$ otherwise. 
Table 1. Re-Optimized $p_{i}^{*}$ Parameters and Radil Used for SASA Computation ACCORding to (HASEl ET AL., 1988)

\begin{tabular}{lll}
\hline Atom type & $p_{i}^{*}$ & $r_{i}$ \\
\hline $\mathrm{CH}\left(\mathrm{sp}^{3}\right)$ & 0.416 & 2.0 \\
$\mathrm{O}\left(\mathrm{sp}^{2}\right)$ & 0.507 & 1.4 \\
$\mathrm{CH}_{2}\left(\mathrm{sp}^{3}\right)$ & 0.472 & 2.0 \\
$\mathrm{O}^{-}$ & 0.577 & 1.4 \\
$\mathrm{CH}_{3}\left(\mathrm{sp}^{3}\right)$ & 0.544 & 2.0 \\
$\mathrm{~N}\left(\mathrm{sp}^{3}\right)$ & 0.620 & 1.5 \\
$\mathrm{C}\left(\mathrm{sp}^{2}\right)$ & 0.356 & 1.5 \\
$\mathrm{~N}\left(\mathrm{sp}^{2}\right)$ & 0.411 & 1.5 \\
$\mathrm{CH}\left(\mathrm{sp}^{2}\right)$ & 0.458 & 1.85 \\
$\mathrm{NH},=\mathrm{N}-\left(\mathrm{sp}^{2}\right)$ & 0.411 & 1.5 \\
$\mathrm{O}\left(\mathrm{sp}^{3}\right)$ & 0.518 & 1.4 \\
$\mathrm{~S}$ & 0.568 & 1.85 \\
\hline
\end{tabular}

Radii are due to (1960).

2.1.2. Desolvation model. We combine formulas (12) and (8) to create a parametric, pairwise algorithm for the desolvation energy $\Delta G_{\text {bind,solv }}$ for use in protein docking simulation. In Algorithms 1-3, we outline the formulation and implementation on both CPUs and GPUs. Algorithm 1 describes the basic CPU implementation of the pairwise surface area and desolvation calculation, and Algorithms 2 and 3 describe the GPU implementation. The CPU and GPU versions are close to numerically equivalent, with the anticipated single-precision floating point representation error averaging $2.26 \times 10^{-6}$ over our benchmarking set.

Our algorithm computes the desolvation free energy of a protein complex by combining known atomic solvation parameters with computed solvent accessible surface areas. Profiling of an initial CPU implementation indicates that the majority of processing time is spent computing the SASA. This agrees with the expected computational complexity-the solvent accessibility computation scales as $O\left(n^{2}\right)$ whereas the desolvation sum is $O(n)$.

The problem of accessibility computation time is further compounded when one considers extending the application of the algorithm to search through conformational space for an energetic minimumaccessibility values are dependent on atomic positions, so each conformational change requires a potential re-evaluation of order $n^{2}$. The desire to perform a large number of desolvation calculations motivates our application of hardware acceleration to this problem.

\subsection{CUSA and CUDE implementations}

Here, we present implementation details for the CUDA-based codes, CUSA and CUDE, along with their reference CPU implementations. The CPU implementations perform multicore calculations, while the GPU implementations perform multi-threaded calculations on NVIDIA graphics cards.

2.2.1. CPU implementation. The reference CPU implementation (Algorithm 1) consists of the previously described accessibility computation followed by a loop that uses the computed accessibility to determine the desolvation contribution of each atom. On lines 8 and 15 of Algorithm 1, atom $i$ is assigned its SASA before overlaps-the factor $S_{i}$ defined in formula (6). The overlap factor of atom $j$ with atom $i$ on lines $10,17,23$, and 29 is the $f_{i j}$ defined in (5).

The intra-molecular SASA computations performed in the loops beginning on lines 7 and 14 correspond to the product of $f_{i j}$ terms in formula (8), and the inter-molecular computations performed beginning on lines 21 and 27 correspond to the product of $f_{i k}$ terms in formula (8). The pairwise desolvation contributions due to changes in SASA during binding are summed on lines 35 and 38 of Algorithm 1.

Our CPU implementation used function inlining where appropriate, avoided string lookups, and tried to maximize re-use of commonly computed values. This implementation did not undergo additional optimization, such as intrinsic hints to reduce L2 cache misses or loop vectorization.

2.2.2. CUDA overview. Modern GPUs are cooperative processing platforms designed for the rendering of 3D graphics, an application that demands high-throughput parallel hardware capable of significant arithmetic density. Originally specialized for high-speed performance in specific graphics applications, the 
Algorithm 1 CPU desolvation

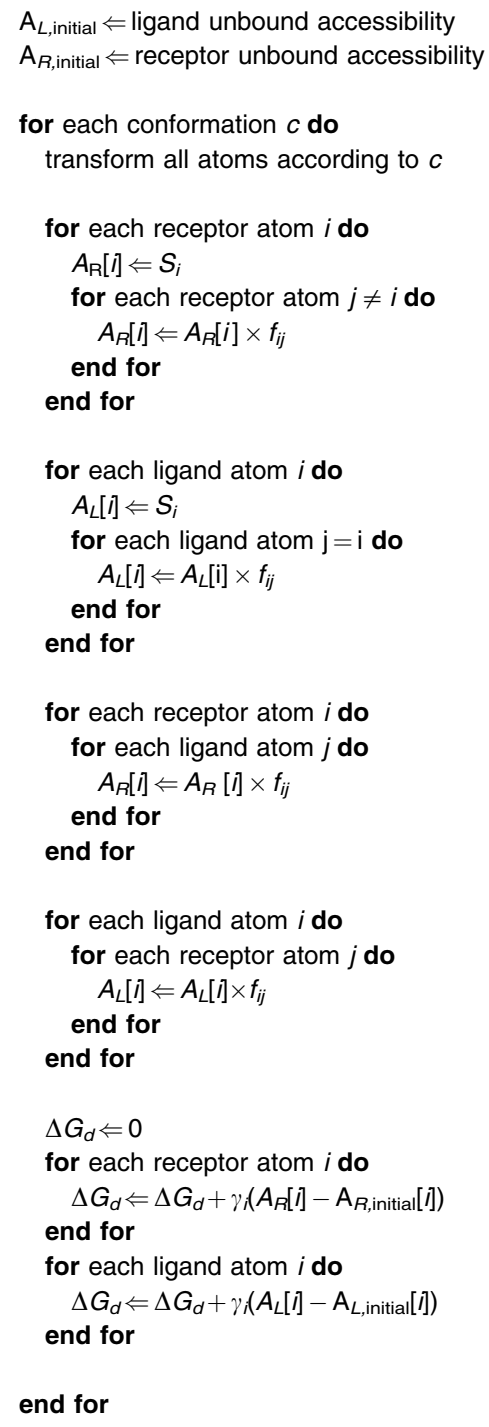

Algorithm 2 CUSA: CPU control code

1: GPU call: calculate unbound ligand accessibility

2: GPU call: calculate unbound receptor accessibility

3: for each conformation $c$ do

GPU call: transform ligand

GPU call: calculate all-complex accessibility

read accessibility from GPU memory

compute desolvation for all atoms

end for

Algorithm 3 CUSA: GPU accessibility thread

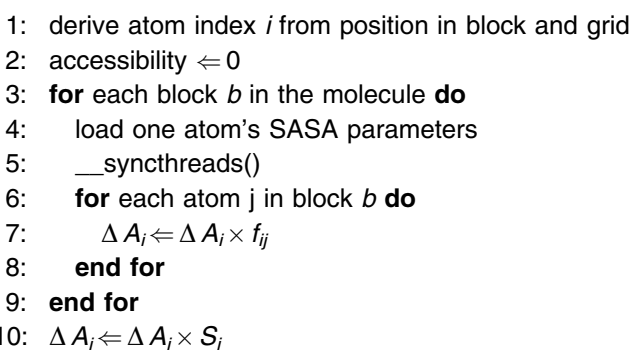


modern GPU strikes a balance between maintaining a somewhat specialized high-speed architecture and exposing enough functionality to become a viable platform for general purpose scientific computing. For a more detailed description of the evolution of GPU programming interfaces, see Owens et al. (2007).

GPUs are high performance devices which, for certain categories of parallelizable problems, can greatly outperform a CPU. This is possible because of the data-parallel nature of the GPU's memory and arithmetic unit layout. A deep pipeline and scores of multi-processors executing uniform calculations allow a GPU to perform computation more frequently and at higher rates than on a general purpose CPU (Owens et al., 2008). With 256 streaming multiprocessors, an NVIDIA GeForce GTX 280 card has a theoretical maximum throughput of 933 GFLOPS, compared with a theoretical maximum throughput of under 50 GFLOPS for an Intel Quad-Core Xeon E5335.

NVIDIA, along with Advanced Micro Devices' ATI division and Intel Corporation are major world-wide suppliers of commodity GPUs. Using a graphics shader language and programming API such as OpenGL or DirectX, one can implement a scientific computing problem on several generations of ATI and NVIDIA cards. However, this approach is cumbersome: it requires a good deal of graphics programming knowledge, authoring multiple versions of shader code to account for generational and manufacturer hardware differences, and forces an unnatural re-interpretation of a problem in the language of 3D rendering.

In 2007, NVIDIA released CUDA (NVIDIA, 2007), a combination hardware/software platform that greatly simplifies the use of commodity GPUs for general purpose scientific computation. Using CUDA, parallel algorithms can be written for and executed on a GPU without any knowledge of graphics programming. CUDA is an NVIDIA product and only runs on modern CUDA-capable NVIDIA GPUs. ATI's Stream Computing (ATI, 2007) initiative plans to provide similar development tools for ATI GPUs, but was not yet fully available at the time this article was written.

On a CUDA-capable GPU, computation is performed by sets of streaming multiprocessors, each containing several compute units. Code is executed as a block of threads on a particular multiprocessor. Blocks of threads are grouped into a grid for distribution onto the GPU. Each multiprocessor contains a small shared memory store that can be accessed by all threads in a given block. Accessing the shared memory store is very cheap-on the order of register access (NVIDIA, 2007). Global memory provides a much larger storage space but is significantly slower to access. Structuring memory access with these capabilities in mind is an important consideration for good utilization of GPU resources.

2.2.3. CUSA and CUDE. Nested computations of order $n^{2}$ are common in scientific computing, and the literature describes several methods of implementing such computations on the GPU (NVIDIA, 2007; Stone et al., 2007; Schive et al., 2008). A common method we adopt is to factor the algorithm to perform $n$ computations in each thread. We then have the GPU compute $n$ of these threads to achieve the necessary $n^{2}$ comparisons. Each singleton thread collects the accessibility contribution from other atoms on a fixed atom.

CUDE (CUDA Desolvation) is our GPU implementation (Algorithm 2). It consists of four device kernel calls which compute accessibility information. First, we compute the prebinding SASA for both proteins. Then, for each conformation we transform the ligand and compute the accessibility of the complex. The algorithm is also capable of transforming the receptor molecule, in order to allow for flexible docking applications.

Our algorithm requires several parameters for each atom, and the parameter set for a reasonably sized molecule will not entirely fit into shared memory. Our device kernel (Algorithm 3) addresses this by dividing the molecule into blocks that are processed in parallel. Furthermore, to minimize the number of reads to global memory, we adopt an iteration pattern suggested by Schive et al. (2008). In their work on pairwise astrophysical systems, they suggest a method of block-based iteration to reduce global memory access. In this method, the threads in a given execution block iterate over the complex atoms one block-size chunk at a time. This allows the threads on a given multiprocessor to cooperatively load the the relevant data for one block's worth of atoms into shared memory. Each thread only reads one atom's parameter set from global memory and relies on the other threads in the block to provide the rest of the data. After the return of the synchronization call on line 5 of Algorithm 3, the multiprocessor's shared memory bank will contain all the atomic parameters needed by each thread in the current iteration. Furthermore, since threads within a block read parameter sets in adjacent memory, this access scheme can be coalesced by CUDA into one hardware read per parameter per block-size iteration. 


\section{RESULTS AND DISCUSSION}

We have adapted a method for approximating the solvent-accessible surface area developed by Hasel et al. (1988) to a form suitable for parallel evaluation on graphics processing units. As an application of our method, we compute desolvation free energies using the ASP model of Eisenberg and McLachlan (1986).

We determined that CUDE, our GPU implementation, substantially outperforms our CPU version. Because the most computationally intensive part of CUDE is the solvent accessibility calculation, similar performance can be expected for CUSA. On average, our GPU based codes compute a given batch of desolvation energies two orders of magnitude faster than our CPU based code. Thus, GPU computation is a valuable tool for decreasing the runtime of our computational biology codes.

\subsection{Model accuracy}

Following the parameter fitting method described in Section 2.1.1 we derived the $p_{i}$ values necessary for the SASA computation using Pauling united atom radii (Pauling, 1960). Table 1 lists our new $p_{i}$ parameters along with the radii used for each atomic group. Compared against the buried area computed with NACCESS (Hubbard et al., 1993), our optimized $p_{i}$ values yield a correlation of 0.95 with a relative error of $9.7 \%$. This correlation was computed over the Mintseris et. al. protein docking benchmark (Mintseris et al., 2005) and is displayed in Figure 1. We withheld 9 randomly selected complexes for use as a test set, leaving the rest as a training set. The correlation on the withheld complexes was 0.96 with a relative error of $9.5 \%$.

As an initial application of our CUSA algorithm, we implemented CUDE, an ASP-based desolvation model and compared with results presented by Weng et al. (1997), which we refer to as the WDV model. This work presents a comparison of experimental and computed free energies for 15 complexes, and lists ASP-computed $\Delta G_{d}$ 's. We noted that on the WDV benchmark, our computed $\Delta G_{d}$ values seem to systematically underestimate the WDV $\Delta G_{d}$ values proportionally to the surface area buried during binding. This suggests that we can compute a correction factor based on the ratio of error in $\Delta G_{d}$ and the buried surface area. Using a five-fold cross validation we trained a correction term $\varepsilon=5.8376 \times \mathrm{BSA}$. Adding $\varepsilon$ to our desolvation energies (formula 12) yields a relative error of $9.1 \%$. The average relative error on the withheld bins was $9.4 \%$ and the individual correction factors for each subset were comparable with the value $\varepsilon$ given above. These relative errors are similar to those observed in other solvation energy models (Zhang et al., 1997).

\subsection{Computational runtime}

CUDE, described in Section 2.2.3, substantially outperforms our reference CPU implementation. Because the dominant cost of running CUDE is the solvent accessibility calculation, speedup results for CUSA are apt to be similar.

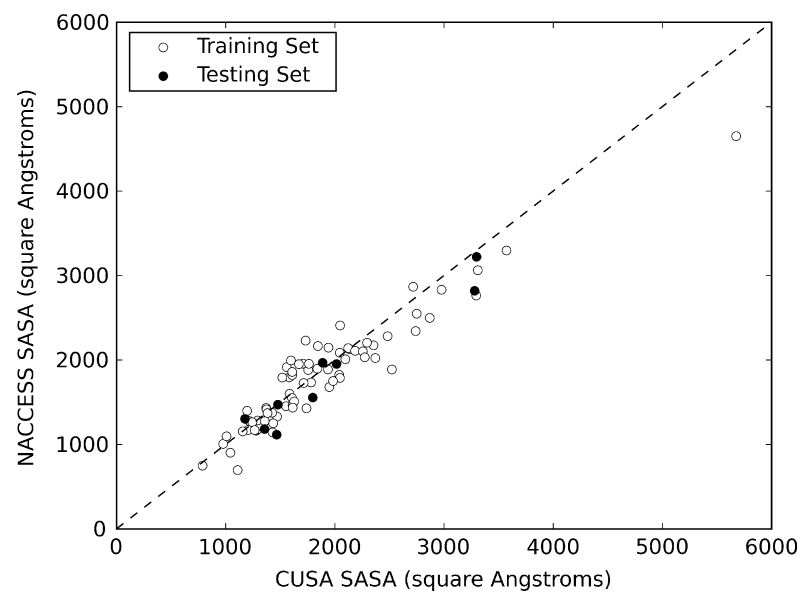

FIG. 1. Buried area correlation between CUSA and NACCESS. 
Table 2. Timing Benchmark Set

\begin{tabular}{|c|c|c|c|}
\hline Large complex & $1 \mathrm{I} 9 \mathrm{R}$ & 1DE4 & $1 \mathrm{~K} 5 \mathrm{D}$ \\
\hline Large receptor & $1 \mathrm{KXQ}$ & $1 \mathrm{BVN}$ & $1 \mathrm{MAH}$ \\
\hline Large ligand & $1 \mathrm{I} 2 \mathrm{M}$ & $1 \mathrm{H} 1 \mathrm{~V}$ & $1 \mathrm{~B} 6 \mathrm{C}$ \\
\hline Average complex & 1JPS & 1FSK & 1DQJ \\
\hline Average receptor & 1BUH & $1 \mathrm{E} 6 \mathrm{E}$ & $1 \mathrm{~A} 2 \mathrm{~K}$ \\
\hline Average ligand & $1 \mathrm{M} 10$ & 1IB1 & $1 \mathrm{FC} 2$ \\
\hline Small complex & 1QA9 & $1 \mathrm{AY} 7$ & 1CGI \\
\hline Small receptor & $1 \mathrm{EZU}$ & 1D6R & $1 \mathrm{AVX}$ \\
\hline Small ligand & 1UDI & 1TMQ & 2SNI \\
\hline
\end{tabular}

Timing numbers were computed over a protein set selected from the Mintseris et al. (2005) protein docking benchmark. To create our timing benchmark, we randomly selected 3 complexes in each of 9 categories: large complex, large ligand, large receptor, average complex, average ligand, average receptor, small complex, small ligand, and small receptor. The selection criteria for the categories was based on the number of atoms in the complex, ligand, and receptor, respectively. Complexes, ligands or receptors containing a number of atoms greater than one-half a standard deviation above the mean for the entire benchmark set were eligible to be selected into the large categories. Similarly, proteins within one-half a standard deviation of the mean were eligible for selection into average categories, and proteins below onehalf of a standard deviation of the mean were eligible for selection into the small categories.

Table 2 lists the PDB identifiers (Berman et al., 2000) of the proteins selected for timing analysis. To simulate a minimization application, we evaluate desolvation energies for each of the complexes for 2000 , $4000,6000,8000$, and 10,000 randomly generated rigid body transformations of the ligand. The runtime reported is the wall-clock time averaged across three runs. For both the CPU implementation and the GPU implementation the measured runtime includes transforming the ligand and receptor, computing the SASA, and finally summing the atomic desolvation contributions. Additionally, both the CPU and GPU runtime numbers reported include relevant set-up such as memory allocation, system threading overhead, and data transfer to the GPU. For more information, please see our source code, available at http://www.mitchell-lab.org.

We compared three versions of the desolvation algorithm designated 2CPU, $8 \mathrm{CPU}$ and $2 \mathrm{GPU}$. The $2 \mathrm{CPU}$ and $8 \mathrm{CPU}$ algorithm split the ligand conformations into two and eight CPU threads respectively. The 2GPU version split the ligand conformations into two CPU system threads, each one claiming one of the machine's GPUs. Our benchmark machine, listed in Table 3, contains a quad-core processor. Because of this, our 8CPU timings are CPU-limited and represent a "maximum load" case.

Table 4 contains the results of our timing analysis. For each complex in our timing benchmark we report 3 -run averaged wall-clock times for 2000, 4000, 6000, 8000, and 10,000 ligand conformations. Additionally, for each complex we report the average speedup factor computed as the average of the speedup $\frac{2 \mathrm{CPU}}{2 \mathrm{GPU}}$ and $\frac{8 \mathrm{CPU}}{2 \mathrm{GPU}}$ for each set of transformations.

Our CUDE implementation substantially outperformed both of our CPU implementations. Because of the $O\left(n^{2}\right)$ complexity of the SASA calculation, the degree of improvement is correlated to the size of the complex -in general, larger complexes show greater speedup. Figure 2 shows a graph of average runtime over all transformation numbers versus complex size. Observed average runtime improvements range from $30 \times(2 \mathrm{GPU}$ vs. $8 \mathrm{CPU}$ ) for $1 \mathrm{AY} 7$, a 1466 -atom complex, to $378 \times(2 \mathrm{GPU}$ vs. $2 \mathrm{CPU})$ for $1 \mathrm{DE} 4$, a 13,107 -atom complex. In addition, the scaling results suggest that the use of careful massively-threaded calculations not only reduces the overall runtime considerably, but it also appears to decrease the effective order of the calculation, as witnessed by the different slope exhibited by the data points for the GPU timings in Figure 2 compared with those taken for calculations on the CPU.

TABLE 3. HARDWARE SPECIFICATIONS

\begin{tabular}{ll}
\hline CPU & Quad-core Intel Xeon E5335, 2.0 GHz \\
RAM & $8,169,904 \mathrm{kB}$ \\
GPU & $2 \times$ NVIDIA GeForce GTX 280 \\
\hline
\end{tabular}


Table 4. Timing Benchmark Results

\begin{tabular}{|c|c|c|c|c|c|c|c|c|c|}
\hline & \multirow[b]{3}{*}{ PDB name } & \multirow[b]{3}{*}{ Complex size } & \multicolumn{5}{|c|}{ Timings for: $\begin{aligned} & 2 C P U \\
& 8 C P U \\
& 2 G P U\end{aligned}$} & \multirow{2}{*}{\multicolumn{2}{|c|}{ Average speedup }} \\
\hline & & & \multicolumn{5}{|c|}{ Number of energy evaluations } & & \\
\hline & & & 2000 & 4000 & 6000 & 8000 & 10000 & $\frac{2 \mathrm{CPU}}{2 \mathrm{GPU}}$ & $\frac{8 \mathrm{CPU}}{2 \mathrm{GPU}}$ \\
\hline \multirow{9}{*}{ Large complex } & $1 \mathrm{I} 9 \mathrm{R}$ & 6573 & 1410 & 2818 & 4226 & 5626 & 7045 & 289 & 145 \\
\hline & & & 705 & 1409 & 2113 & 2818 & 3521 & & \\
\hline & & & 6 & 10 & 15 & 19 & 24 & & \\
\hline & 1DE4 & 13107 & 5570 & 11136 & 16697 & 22229 & 27801 & 378 & 189 \\
\hline & & & 2786 & 5568 & 8352 & 11130 & 13911 & & \\
\hline & & & 16 & 30 & 44 & 59 & 73 & & \\
\hline & $1 \mathrm{~K} 5 \mathrm{D}$ & 5566 & 1004 & 2009 & 3013 & 4020 & 5024 & 271 & 136 \\
\hline & & & 504 & 1007 & 1509 & 2011 & 2513 & & \\
\hline & & & 5 & 8 & 11 & 15 & 18 & & \\
\hline \multirow{9}{*}{ Large receptor } & $1 \mathrm{KXQ}$ & 4826 & 758 & 1515 & 2272 & 3029 & 3791 & 229 & 115 \\
\hline & & & 380 & 759 & 1138 & 1517 & 1897 & & \\
\hline & & & 4 & 7 & 10 & 13 & 16 & & \\
\hline & $1 \mathrm{BVN}$ & 4443 & 643 & 1285 & 1927 & 2569 & 3214 & 210 & 105 \\
\hline & & & 323 & 644 & 965 & 1286 & 1607 & & \\
\hline & & & 4 & 7 & 9 & 12 & 15 & & \\
\hline & 1MAH & 4576 & 685 & 1367 & 2053 & 2737 & 3420 & 219 & 109 \\
\hline & & & 343 & 685 & 1026 & 1369 & 1710 & & \\
\hline & & & 4 & 7 & 10 & 13 & 15 & & \\
\hline \multirow{9}{*}{ Large ligand } & $1 \mathrm{I} 2 \mathrm{M}$ & 4245 & 591 & 1180 & 1770 & 2360 & 2948 & 198 & 99 \\
\hline & & & 296 & 591 & 886 & 1181 & 1476 & & \\
\hline & & & 4 & 7 & 9 & 12 & 15 & & \\
\hline & $1 \mathrm{H} 1 \mathrm{~V}$ & 5414 & 954 & 1907 & 2859 & 3810 & 4762 & 268 & 134 \\
\hline & & & 477 & 953 & 1430 & 1906 & 2382 & & \\
\hline & & & 4 & 8 & 11 & 14 & 18 & & \\
\hline & $1 \mathrm{~B} 6 \mathrm{C}$ & 3433 & 386 & 771 & 1156 & 1541 & 1922 & 174 & 87 \\
\hline & & & 193 & 386 & 578 & 770 & 963 & & \\
\hline & & & 3 & 5 & 7 & 9 & 11 & & \\
\hline \multirow{9}{*}{ Average complex } & 1JPS & 4858 & 769 & 1536 & 2303 & 3073 & 3837 & 234 & 117 \\
\hline & & & 385 & 769 & 1154 & 1538 & 1922 & & \\
\hline & & & 4 & 7 & 10 & 13 & 16 & & \\
\hline & 1FSK & 4577 & 683 & 1366 & 2052 & 2732 & 3410 & 218 & 109 \\
\hline & & & 342 & 684 & 1025 & 1367 & 1708 & & \\
\hline & & & 4 & 7 & 10 & 13 & 15 & & \\
\hline & 1DQJ & 4245 & 590 & 1177 & 1768 & 2355 & 2941 & 202 & 101 \\
\hline & & & 295 & 589 & 884 & 1178 & 1472 & & \\
\hline & & & 4 & 6 & 9 & 12 & 14 & & \\
\hline \multirow{9}{*}{ Average receptor } & $1 \mathrm{BUH}$ & 2916 & 278 & 555 & 832 & 1109 & 1386 & 139 & 70 \\
\hline & & & 139 & 278 & 416 & 555 & 693 & & \\
\hline & & & 3 & 5 & 6 & 8 & 10 & & \\
\hline & $1 \mathrm{E} 6 \mathrm{E}$ & 4377 & 625 & 1250 & 1873 & 2497 & 3123 & 211 & 105 \\
\hline & & & 313 & 625 & 937 & 1250 & 1562 & & \\
\hline & & & 4 & 7 & 9 & 12 & 15 & & \\
\hline & $1 \mathrm{~A} 2 \mathrm{~K}$ & 3560 & 413 & 827 & 1240 & 1650 & 2066 & 178 & 89 \\
\hline & & & 207 & 414 & 620 & 827 & 1033 & & \\
\hline & & & 3 & 5 & 7 & 9 & 11 & & \\
\hline
\end{tabular}


TABle 4. Continued

\begin{tabular}{|c|c|c|c|c|c|c|c|c|c|}
\hline & \multirow[b]{3}{*}{ PDB name } & \multirow[b]{3}{*}{ Complex size } & \multicolumn{5}{|c|}{ Timings for: $\begin{aligned} & 2 C P U \\
& 8 C P U \\
& 2 G P U\end{aligned}$} & \multirow{2}{*}{\multicolumn{2}{|c|}{ Average speedup }} \\
\hline & & & \multicolumn{5}{|c|}{ Number of energy evaluations } & & \\
\hline & & & 2000 & 4000 & 6000 & 8000 & 10000 & $\frac{2 \mathrm{CPU}}{2 \mathrm{GPU}}$ & $\frac{8 \mathrm{CPU}}{2 \mathrm{GPU}}$ \\
\hline \multirow{9}{*}{ Average ligand } & $1 \mathrm{M} 10$ & 3688 & 445 & 888 & 1333 & 1776 & 2216 & 187 & 93 \\
\hline & & & 223 & 444 & 666 & 888 & 1111 & & \\
\hline & & & 3 & 5 & 7 & 10 & 12 & & \\
\hline & 1IB1 & 5046 & 826 & 1650 & 2476 & 3305 & 4124 & 248 & 124 \\
\hline & & & 414 & 827 & 1240 & 1652 & 2065 & & \\
\hline & & & 4 & 7 & 10 & 13 & 16 & & \\
\hline & $1 \mathrm{FC} 2$ & 2010 & 133 & 264 & 396 & 528 & 661 & 90 & 45 \\
\hline & & & 67 & 133 & 199 & 265 & 331 & & \\
\hline & & & 2 & 4 & 5 & 6 & 7 & & \\
\hline \multirow{9}{*}{ Small complex } & 1QA9 & 1622 & 87 & 173 & 259 & 345 & 430 & 68 & 34 \\
\hline & & & 44 & 87 & 130 & 172 & 215 & & \\
\hline & & & 2 & 3 & 4 & 5 & 6 & & \\
\hline & $1 \mathrm{AY} 7$ & 1466 & 71 & 142 & 213 & 284 & 354 & 59 & 30 \\
\hline & & & 36 & 71 & 107 & 142 & 178 & & \\
\hline & & & 2 & 3 & 4 & 5 & 6 & & \\
\hline & 1CGI & 2239 & 166 & 331 & 496 & 661 & 826 & 101 & 51 \\
\hline & & & 83 & 166 & 248 & 331 & 413 & & \\
\hline & & & 3 & 4 & 5 & 7 & 8 & & \\
\hline \multirow{9}{*}{ Small receptor } & $1 \mathrm{EZU}$ & 3854 & 485 & 969 & 1454 & 1938 & 2421 & 182 & 91 \\
\hline & & & 243 & 485 & 727 & 969 & 1211 & & \\
\hline & & & 4 & 6 & 8 & 11 & 13 & & \\
\hline & 1D6R & 2056 & 140 & 279 & 418 & 557 & 697 & 91 & 46 \\
\hline & & & 70 & 140 & 210 & 279 & 349 & & \\
\hline & & & 2 & 4 & 5 & 6 & 7 & & \\
\hline & $1 \mathrm{AVX}$ & 2916 & 280 & 559 & 838 & 1116 & 1395 & 141 & 71 \\
\hline & & & 140 & 280 & 420 & 559 & 699 & & \\
\hline & & & 3 & 5 & 6 & 8 & 10 & & \\
\hline \multirow{9}{*}{ Small ligand } & 1UDI & 2472 & 200 & 400 & 600 & 801 & 1000 & 115 & 58 \\
\hline & & & 101 & 200 & 300 & 400 & 500 & & \\
\hline & & & 3 & 4 & 6 & 7 & 8 & & \\
\hline & 1TMQ & 4479 & 657 & 1312 & 1968 & 2623 & 3280 & 216 & 108 \\
\hline & & & 329 & 657 & 984 & 1312 & 1640 & & \\
\hline & & & 4 & 7 & 9 & 12 & 15 & & \\
\hline & $2 \mathrm{SNI}$ & 2451 & 199 & 398 & 597 & 796 & 995 & 111 & 55 \\
\hline & & & 100 & 199 & 299 & 398 & 497 & & \\
\hline & & & 3 & 4 & 6 & 7 & 9 & & \\
\hline
\end{tabular}

\subsection{GPU speedup analysis}

Neither the CPU nor CUDE GPU implementations received extensive optimization. Both implementations are straightforward, although implementing CUDE certainly required more care to avoid performance pitfalls. Several goals can be summarized to help achieve good GPU performance with CUDA:

1. Factoring of the algorithm to maximize arithmetic operations on values stored in shared memory

2. Avoiding use of global memory in inner device kernel loops

3. Ensuring that global memory reads are coalesced when possible

4. Avoiding conditional statements that cause divergent thread execution pathways

5. Avoiding hardware-specific performance pitfalls described in (NVIDIA 2007), such as the mod operator 


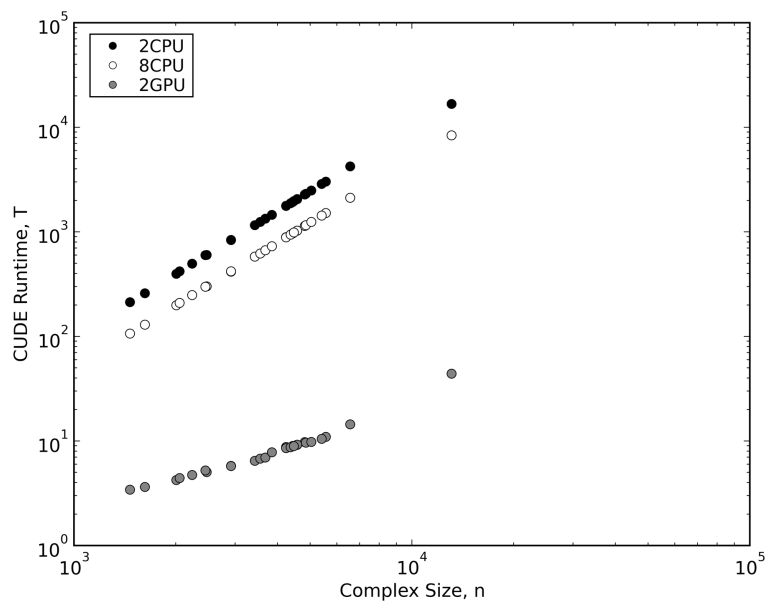

FIG. 2. Average runtime versus complex size.

Goal 1 is arguably the most important from a high level perspective. Algorithms that will see the greatest gain from GPU computation are parallelizable algorithms that perform a high number of arithmetic operations for each memory access. Algorithms that require more frequent access to global memory can still see good performance benefits but will require more effort to optimize. The remaining goals can be dealt with to a large extent during implementation. All are described in greater detail in (NVIDIA, 2007).

Careful analysis of a given problem is vital before pursuing a GPU implementation. The best problems will be those that can be implemented using many arithmetic computations for relatively infrequent memory access. In theory, the best performing CUDA algorithm would be one that does arithmetic computation without ever reading or writing to memory. Although CUDA has some capability to hide memory latency by performing independent instructions while memory requests complete, global memory access is slow and should be minimized. Consequently, a significant determining factor of the speedup of a GPU algorithm is achieving balance in the amount of data required for each individual algorithmic computation.

In our case, each pairwise desolvation contribution only depends on a small number of parameters, 24 bytes per atom. This allows many atoms to fit into shared memory and minimizes how often data needs to be read from global memory. Simultaneously, our algorithm has high enough arithmetic density to amortize the cost of inevitable global memory access.

Algorithms that perform simple calculations on large amounts of non-coherent data are less optimal candidates for GPU implementation, but can still yield speed ups with appropriate optimization. For example, we implemented a version of the popular DFIRE statistical potential (Zhou et al., 2002) for the GPU and observed a much more modest speedup. We believe this is likely due to the model's large number of parameters describing interactions between atom/residue-atom/residue pairs.

\section{ACKNOWLEDGMENTS}

We would like to thank John Stone for his help on performance metrics and understanding GPU fundamentals, Kurt Richards for his help with diagrams, and Nancy Horton, Nathan Baker, Mark Harris, and John Owens for their correspondence regarding their work. This research was funded by the U.S. Department of Energy Genomics: GTL and SciDAC Programs (DE-FG02-04ER25627). In addition, we thank NVIDIA for providing us with two GTX 280 graphics cards through their Professor Partnership Program.

\section{DISCLOSURE STATEMENT}

No competing financial interests exist. 


\section{REFERENCES}

ATI. 2007. Advanced Micro Devices Inc. AMD stream computing. http://ati.amd.com/technology/streamcomputing/ index.html. Available at: Accessed February 1, 2009.

Berman, H., Westbrook, J., Feng, Z., et al. 2000. The Protein Data Bank. Nucleic acids Res. 28, 235-242.

Buck, I., Foley, T., Horn, D., et al. 2004. Brook for GPUs: stream computing on graphics hardware, 777-786. In: SIGGRAPH '04: ACM SIGGRAPH 2004 Papers. ACM, New York.

Caflisch, A., Niederer, P., and Anliker, M. 1992. Monte Carlo docking of oligopeptides to proteins. Proteins 13, $223-230$.

Camacho, C.J., Weng, Z., Vajda, S., et al. 1999. Free energy landscapes of encounter complexes in protein-protein association. Biophys. J. 76, 1166-1178.

Connolly, M.L. 1983. Analytical molecular surface calculation. J. Appl. Cryst. 16, 548-558.

Cummings, M.D., Hart, T.N., and Read, R.J. 1995. Atomic solvation parameters in the analysis of protein-protein docking results. Protein Sci. 4, 2087-2099.

Eisenberg, D., and McLachlan, A.D. 1986. Solvation energy in protein folding and binding. Nature 319, 199-203.

Eisenberg, D., Wesson, M., and Yamashita, M. 1989. Interpretation of protein folding and binding with atomic solvation parameters. Chem. Src. 29A, 217-211.

Fauchere, J.L., and Pliska, V. 1983. Hydrophobic parameters of amino acid side chains from the partitioning of nacetyl-amino acid amides. Eur. J. Med. Chem. 18, 369-375.

Fraczkiewicz, R., and Braun, W. 1998. Exact and efficient analytical calculation of the accessible surface areas and their gradients for macromolecules. J. Comput. Chem. 19, 319-333.

Given, J.A., and Gilson, M.K. 1998. A hierarchical method for generating low-energy conformers of a protein-ligand complex. Proteins 33, 475-495.

Goodsell, D.S., and Olson, A.J. 1990. Automated docking of substrates to proteins by simulated annealing. Proteins 8 , 195-202.

Gray, J.J., Moughan, S.E., Wang, C., et al. 2003. Protein-protein docking with simultaneous optimization of rigid body displacement and side chain conformations. J. Mol. Biol. 331, 281-299.

Hart, T.N., and Read, R.J. 1992. A multiple-start Monte Carlo docking method. Proteins 13, 206-222.

Hasel, W., Hendrickson, T.F., and Still, W.C. 1988. A rapid approximation to the solvent accessible surface areas of atoms. Tetrahedron Comput. Methodol. 1, 103-116.

Horton, N., and Lewis, M. 1992. Calculation of the free energy of association for protein complexes. Protein Sci. 1, 169-181.

Hubbard, S., and Thorton, J. 1993. NACCESS [Computer Program] Available at: www.bioinf.manchester.ac.uk/ naccess/. Accessed February 1, 2009.

Juffer, A.H., Eisenhaber, F., Hubbard, S.J., et al. 1995. Comparison of atomic solvation parametric sets: applicability and limitations in protein folding and binding. Protein Sci. 4, 2499-2509.

Klepeis, J.L., Ierapetritou, M.G., and Floudas, C.A. 1998. Protein folding and peptide docking: a molecular modeling and global optimization approach. Comp. Chem. Eng. 22, S3-S10.

Krüger, J., and Westermann, R. 2005. Linear algebra operators for GPU implementation of numerical algorithms, 234. In: SIGGRAPH '05: ACM SIGGRAPH 2005 Courses. ACM, New York.

Lee, B., and Richards, F.M. 1971. The interpretation of protein structures: estimation of static accessibility. J. Mol. Biol. 55, 379-400.

Lesser, G.J., and Rose, G.D. 1990. Hydrophobicity of amino acid subgroups in proteins. Proteins 8, 6-13.

Marcia, R.F., Mitchell, J.C., and Rosen, J.B. 2005. Iterative convex quadratic approximation for global optimization in protein docking. Comput. Optim. Appl. 32, 285-297.

Mintseris, J., Wiehe, K., Pierce, B., et al. 2005. Protein-protein docking benchmark 2.0: an update. Proteins 60, 214-216.

Mitchell, J.C., Phillips, A.T., Rosen, J.B., et al. 1999. Coupled optimization in protein docking, 180-184. In: Istrail, S., et al., eds. RECOMB 99. ACM Press, New York.

NVIDIA Corp. 2007. NVIDIA CUDA (Compute Unified Device Architecture) Programming Guide, Version 1.0.

Owens, J.D., Luebke, D., Govindaraju, N., et al. 2007. A survey of general-purpose computation on graphics hardware. Comput. Graphics Forum 26, 80-113.

Owens, J.D., Houston, M., Luebke, D., et al. 2008. GPU computing. Proc. IEEE 08.

Pauling, L. 1960. The Nature of the Chemical Bond, 3rd ed. Cornell University Press, Ithaca, NY.

Radzicka, A., and Wolfenden, R. 1988. Comparing the polarities of the amino acids: side-chain distribution coefficients between the vapor phase, cyclohexane, 1-octanol, and neutral aqueous solution. Biochemistry 27, 1664-1670.

Radzicka, A., Young, G., and Wolfenden, R. 1993. Lack of water transport by amino acid side chains or peptides entering a nonpolar environment. Biochemistry 32, 6807-6809.

Rarey, M., Kramer, B., Lengauer, T., et al. 1996. A fast flexible docking method using an incremental construction algorithm. J. Mol. Biol. 261, 470-489. 
Sanner, M., Olson, A.J., and Spehner, J.C. 1996. Reduced surface: an efficient way to compute molecular surfaces. Biopolymers 38, 305-320.

Schive, H.-Y., Chien, C.-H., Wong, S.-K., et al. 2008. Graphic-card cluster for astrophysics (graCCA)—performance tests. New Astron. 13, 418-435.

Shrake, A., and Rupley, J. 1973. Environment and exposure to solvent of protein atoms. Lysozyme and insulin. J. Mol. Biol. 79, 351-371.

Stone, J.E., Phillips, J.C., Freddolino, P.L., et al. 2007. Accelerating molecular modeling applications with graphics processors. J. Comput. Chem. 28, 2618-2640.

Totrov, M., and Abagyan, R. 1997. Flexible protien-ligand docking by global energy optimization in internal coordinates. Proteins S1, 215-220.

Wagoner, J.A., and Baker, N.A. 2006. Assessing implicit models for nonpolar mean solvation forces: the importance of dispersion and volume terms. Proc. Natl. Acad. Sci. USA 103, 8331-8336.

Weiser, J., Shenkin, P.S., and Still, W. C. 1999. Approximate atomic surfaces from linear combinations of pairwise overlaps (lcpo). J. Comput. Chem. 20, 217-230.

Weiser, J., Shenkin, P.S., and Still, W.C. 1999. Fast, approximate algorithm for detection of solvent-inaccessible atoms. J. Comput. Chem. 20, 586-596.

Weiser, J., Shenkin, P.S., and Still, W.C. 1999. Approximate solvent-accessible surface areas from tetrahedrally directed neighbor densities. Biopolymers 50, 373-380.

Weng, Z., Delisi, C., and Vajda, S. 1997. Empirical free energy calculation: comparison to calorimetric data. Protein Sci. 6, 1976-1984.

Wesson, L., and Eisenberg, D. 1992. Atomic solvation parameters applied to molecular dynamics of proteins in solution. Protein Sci. 1, 227-235.

Yang, J., Wang, Y., and Chen, Y. 2007. GPU accelerated molecular dynamics simulation of thermal conductivities. J. Comput. Phys. 221, 799-804.

Zhang, C., Vasmatzis, G., Cornette, J.L., et al. 1997. Determination of atomic desolvation energies from the structures of crystallized proteins. J. Mol. Biol. 267, 707-726.

Zhou, H., and Zhou, Y. 2002a. Stability scale and atomic solvation parameters extracted from 1023 mutation experiments. Proteins 49, 483-492.

Zhou, H., and Zhou, Y. 2002b. Distance-scaled, finite ideal-gas reference state improves structure-derived potentials of mean force for structure selection and stability prediction. Protein Sci. 11, 2714-2726.

Address reprint requests to:

Dr. Julie C. Mitchell

Departments of Mathematics and Biochemistry

University of Wisconsin-Madison

480 Lincoln Drive

Madison, WI 53706

E-mail: jcmitchell@wisc.edu 
